\title{
Beyond Bone: Micro-Computed Tomography for Preclinical Specimen Imaging and Applications for Soft Tissue Discovery and Translational Therapeutic Testing
}

\author{
Sergio X. Vasquez, Dan Small, and David Weinstein
}

Numira Biosciences, Salt Lake City, UT USA

Historically, micro-computed tomography (microCT) for preclinical biological specimens has been constrained to high-fidelity imaging of bone or other similarly dense tissues [1]. On the contrary, noncontrast enhanced microCT imaging provides rough visual approximations of soft tissues such as fat, muscle, and lung, but finer details are not readily observed under routine platform parameters. The advent of contrast enhanced processing of ex-vivo specimens introduced an increase in the number of viable soft tissue applications amenable to high resolution microCT imaging and analysis techniques [2]. Additionally, vascular casting with radiopaque polymers has increased the awareness of microCT utility in preclinical therapeutic oncology studies of anti-angiogenic efficacy [3,4]. Furthermore, while intensive research and development in specimen processing methods has yielded a number of intriguing qualitative and quantitative soft-tissue endpoints [5], some applications have presented as potentially more useful in analysis of translational drug development, especially where existing technologies have failed to allow for more comprehensive elucidation of new and ongoing therapeutic strategies, including imaging and analysis of pulmonary fibrosis [6]. For these reasons, though microCT is now considered the gold standard for quantitative microstructural bone analysis [7], microCT adoption for preclinical soft-tissue studies is on course for becoming accepted as a routine endpoint standard.

Preclinical microCT studies of bone have been well documented with morphological observation and bone mineral density analysis (BMD) already accepted as the gold standard for assessing deviation from normal biological outcomes in spontaneous, genetic or induced skeletal perturbations. Owing to the high density of bone $(+700-+3000$ on the Hounsfield scale), parallel scanning with soft tissue generally produces reconstructed images of bone only. For those preclinical soft tissues whose densities allow for visualization and disambiguation from bone -- such as fat, muscle or lung - limited whole volume studies have been conducted, without providing much detail highlighting changes in microstructure. More complex quantitative analysis is therefore often constrained exclusively to bone.

To fully exploit the 3-dimensional data derived from microCT imaging, early contrast enhancement studies of whole-mount rodent embryos showed that successfully processed soft-tissue achieved sufficient density for tomographic visualization. Permeability and small size of specimens harvested at gestational and early post-natal timepoints allowed for a rapid processing and imaging methodology which appeared useful for observation of gross morphological changes, atlas generation and digital archiving by microCT. Further studies detailing the processing of adult specimen tissues, including various incubation stains and non-traditional model organisms expanded the application base further. With these discoveries, awareness of microCT as a useful modality to image both bone and soft tissue was promoted.

Meaningful utility of microCT capacity required optimization and progression of both conventional and current knowledge to include translational applications in preclinical drug discovery pipelines. As such, higher-level analysis for bone diseases have been developed, along with non-destructive visualization of cartilage in joints, as well as more comprehensive imaging and analysis of atherosclerotic plaque burden 
in cardiovascular disease. Additionally, realizing the limitations associated with commercially available vascular perfusion reagents and requiring a radiopaque compound more amenable to the tumor microenvironment, a reagent coined AltaBlu Prime ${ }^{\mathrm{TM}}$ was developed to fulfill this purpose.

Of particular interest are those microCT applications recently developed which show great promise in providing a framework by which to image and analyze disease endpoints unachievable by alternative technologies, namely pulmonary fibrosis imaging. Preclinical pulmonary fibrosis can be induced via acute or chronic insult and ideally characterized by inflammatory progression to fibrotic outcome of the lung tissue. Traditionally visualized by stepped histological sectioning, an admittedly limited understanding of the whole-mount lung is achieved, and may inadvertently result in high error-rate in reporting despite cohort size. The reported microCT application includes non-destructive whole-mount imaging and analysis of the harvested lung at iterative timepoints, aiding in the visualization and quantification of fibrosis burden and potential therapeutic response. This data is provided in a digital format, so that the lungs are preserved for further histological, immunohistochemical, or molecular analysis for correlative statistics if desired.

[1] SX Vasquez, et al, Anatomical Record 29 (2008), p. 475-487.

[2] JT Johnson, et al, PLOS Genetics, 2 (2006), e61.

[3] SX Vasquez, et al, PLOS One, 6 (2011), e19099.

[4] F Gao, et al, Integrative Biology, 3 (2011), p. 479-489.

[5] DJ Loyd, et al, PLOS One, 6 (2011), e18800.

[6] O Uitto, et al, World Molecular Imaging Congress (2011).

[7] W Tjong, et al, Medical Physics, 39 (2012), p. 1893-1903.

Figure 1. Representative microCT imaging applications and analysis. Left to right: volume rendering of P0 Mouse Pup Skeleton, soft tissue segmentation of various P0 Mouse Pup organs, surface roughness heat map of diseased adult mouse paw, vessel radius heat map of AltaBlu Prime ${ }^{\mathrm{TM}}$ perfused adult rat brain.
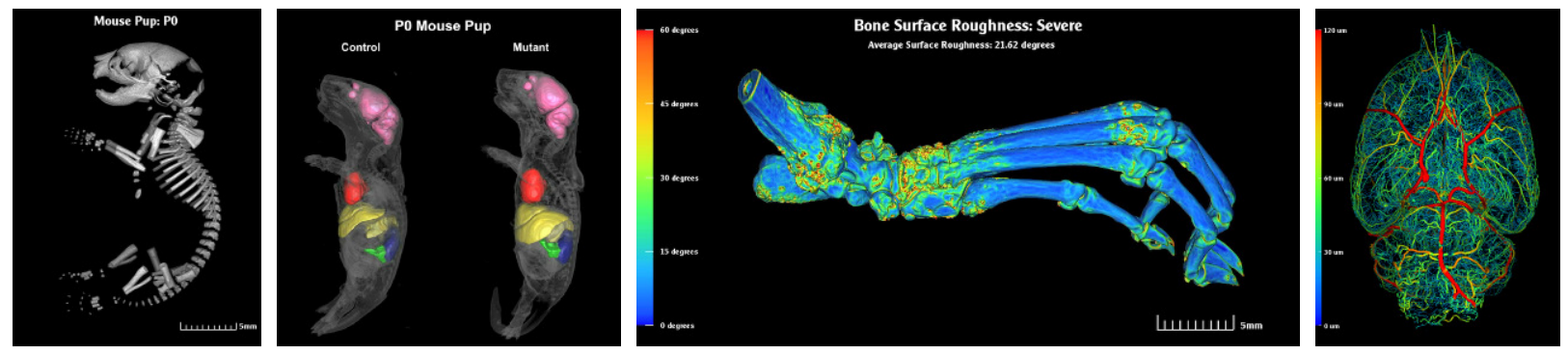

Figure 2. MicroCT imaged mouse lung. Left to right: coronal projection, fibrosis segmentation, histological validation, microCT analysis histogram
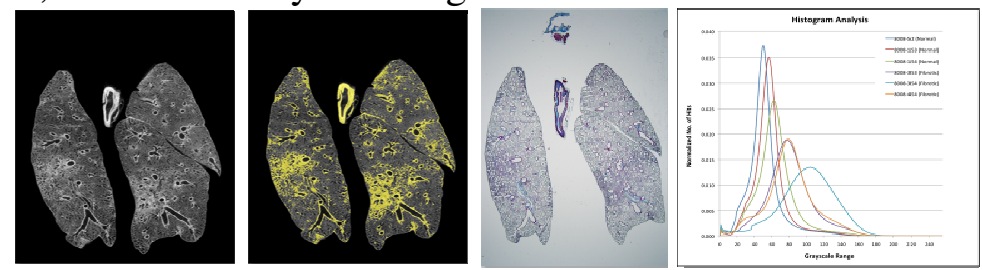